\title{
E1 Huerto Escolar como recurso para iniciar la Alfabetización Ambiental en Educación Infantil
}

\author{
Fátima Rodríguez-Marín (D), María África Portillo Guerrero (D), \\ María Puig Gutiérrez \\ Departamento de Didáctica de las Ciencias Experimentales y Sociales, Facultad Ciencias de la Educación. \\ Universidad de Sevilla.España.frodmar@us.es, afriportillo@gmail.com,mpuig@us.es
}

[Recibido: 4 marzo 2020. Revisado: 12 marzo 2020. Aceptado: 1 febrero 2021]

\begin{abstract}
Resumen: Los graves problemas socioambientales a los que se enfrenta nuestro mundo, deberían ser abordados de manera prioritaria en las propuestas didácticas actuales. De este modo, habría que trabajar en propuestas que incrementasen la capacidad de ajuste de la población a un mundo muy diferente al actual (con menos recursos, pérdida de biodiversidad y cambio climático; en decrecimiento), porque ese es el mundo en el que vivirán quienes hoy están en la etapa de Educación Infantil.

Se presenta así una experiencia realizada en un aula con niños y niñas de 3 años, en la que se ha utilizado el huerto escolar como eje central de una propuesta didáctica basada en el modelo de investigación escolar. Los resultados muestran que este recurso favorece la alfabetización ambiental, al tiempo que sirve para articular propuestas didácticas que contribuyen a la resiliencia de la población.
\end{abstract}

Palabras clave: Educación Infantil, Huertos Escolares, alfabetización ambiental, educación ambiental, experiencias fuera del aula

The school garden as a resource to initiate environmental literacy in childhood education

Abstract: The serious socio-environmental problems facing our world should be addressed as a priority in current educational proposals. In this way, work should be done on proposals that increase the population's capacity to adjust to a world that is very different from today's (with fewer resources, loss of biodiversity and climate change; in degrowth), because that is the world in which the children who are currently in the stage of Childhood Education, will live.

This is an experience carried out in a classroom with three-year-old children, in which the school garden has been used as the central axis of a didactic proposal based on the school research model. The results show that this resource favors environmental literacy, while serving to articulate didactic proposals that contribute to the resilience of the population.

Keywords: Childhood education, School garden, environmental literacy, environmental education, outdoor learning

Para citar este artículo: Rodríguez-Marín F., Portillo Guerrero M. A. y Puig Gutiérrez M. (2021) El Huerto Escolar como recurso para iniciar la Alfabetización Ambiental en Educación Infantil. Revista Eureka sobre Enseñanza y Divulgación de las Ciencias 18(2), 2501. doi: 10.25267/Rev_Eureka_ensen_divulg_cienc.2021.v18.i2.2501

\section{Alfabetización Ambiental en Educación Infantil}

En los ámbitos de la Educación Ambiental (EA, en lo sucesivo) y de la Enseñanza de las Ciencias ya está consolidada la idea de que es necesario formar a la población para tratar y resolver los complejos problemas socioambientales generados por la actividad humana (García 2006). En este sentido, en la etapa de Educación Infantil, tiene una función imprescindible la aproximación inicial de los escolares a las perspectivas científicas sobre la realidad (Cañal 2006) ya que son diversas las investigaciones, que apoyan este acercamiento a la ciencia de los más pequeños (Cañal 2006; Eshach y Fried 2005; Eshach Haim 2006; García-Carmona, Criado y Cañal 2014; Gomez-Montilla y Ruiz-Gallardo 2016; Guzmán-Cruz, García-Carmona y Criado 2017; Mazas y Hervas 2018; Torres-Porras, Alcántara, Arrebola, Rubio y Mora 2017). 
Eshach y Fried (2005) resumen en seis, las razones fundamentales para este acercamiento en edades tempranas: (1) Los niños y niñas disfrutan naturalmente observando y pensando en la naturaleza; (2) La exposición de los estudiantes a la ciencia desarrolla positivamente actitudes hacia la ciencia; (3) Exposición temprana a fenómenos científicos lleva a una mejor comprensión de conceptos científicos estudiados posteriormente; (4) El uso de un lenguaje científicamente informado a temprana edad influye en el desarrollo de conceptos científicos; (5) Los niños y niñas pueden entender conceptos científicos y razonar científicamente; y (6) La ciencia es un medio eficiente para desarrollar pensamiento científico.

Una alfabetización científica que sirva al objetivo del tratamiento educativo de los problemas socioambientales, debe convertirse en alfabetización ambiental. Habiendo diversidad de interpretaciones existe el consenso de que «es aquella alfabetización que comprende una conciencia y una preocupación por el medio ambiente y sus problemas asociados y que requiere los conocimientos, las habilidades y las motivaciones para trabajar en la solución de los problemas ambientales actuales y futuros» (Álvarez-García, Sureda-Negre y Comas-Forgas 2018 p. 119).

\section{Experiencias fuera del aula: Entornos cercanos medio natural y humanizado}

Establecer vínculos con la naturaleza, descubrir las consecuencias que nuestras acciones tienen sobre la misma, desarrollar destrezas y habilidades de cuidado y protección, son objetivos que deben proponerse desde las escuelas en las diferentes etapas educativas.

A edades tempranas, resulta, además, esencial el contacto directo con la naturaleza, más aún en un momento como el actual, en el que es frecuente la disociación entre el mundo natural y la vida diaria de los más pequeños (Fjørtoft 2001, Louv 2005, Palmer 2006). Según Blair (2009), los escolares de hoy carecen de experiencia directa con la complejidad de los ecosistemas naturales. En Estados Unidos, por ejemplo, se calcula que aproximadamente el $83 \%$ de la población vive en áreas metropolitanas. Teniendo en cuenta que el contexto en el que los niños y niñas viven se convierte en el escenario sobre el que desarrollan su imaginación y comprenden el mundo (Mergen, 2003), resulta esencial ofrecer a los más pequeños experiencias de contacto directo con la naturaleza, para que esta entre a formar parte también de ese escenario.

Investigaciones como las realizadas por McCurdy, Winterbottom, Mehta y Roberts (2010), demuestran que la falta de contacto de los niños y niñas con el entorno natural afecta negativamente a la salud, provocando múltiples patologías. De forma paralela, son diversos los estudios que manifiestan las ventajas que el contacto con la naturaleza tiene en los más pequeños. Así, por ejemplo, la tesis doctoral defendida por Collado (2012) evidencia el efecto restaurador de la naturaleza en los menores, al tiempo que destaca como aumentan las actitudes y comportamientos pro-ambientales a través del contacto con el medio natural. Por su parte, The Natural Learning Initiative (NLI) (2012) ofrece un interesante resumen de los principales beneficios de la conexión de los niños y niñas con la naturaleza derivados de múltiples investigaciones, destacando entre ellos los siguientes:

- Favorece el desarrollo integral.

- Potencia la creatividad y la resolución de problemas.

- Mejora las habilidades cognitivas.

- Mejora el rendimiento académico.

- Reduce los síntomas del trastorno por déficit de atención. 
- Incrementa la actividad física.

- Mejora la nutrición.

- Mejora la vista.

- Mejora las relaciones sociales.

- Mejora la autodisciplina.

- Reduce el estrés.

Podemos decir, por lo tanto, que la Educación Infantil es un momento idóneo para establecer ese vínculo con la naturaleza que resulta tan beneficioso a corto y largo plazo (Poppell y Monroe 2017). Aprovechar la capacidad innata de los más pequeños para explorar el mundo que les rodea y acercarlos a su entorno natural, es pues, una manera de comenzar a conectarlos con la naturaleza. Así, desde las escuelas, sería deseable que se apostara por una educación ambiental de calidad, que pusiera al alcance de los más pequeños recursos y estrategias para explorar su entorno, desarrollando experiencias de aprendizaje enriquecedoras que fortalezcan la conexión con la naturaleza (Prince 2010).

\section{Los Huertos Escolares como recurso para enseñar ciencia que promuevan ciudadanos activos}

El Huerto Escolar (HE, en lo sucesivo) puede ser un recurso que ayude a conectar los dos aspectos anteriormente desarrollados en la Educación Infantil. El aprendizaje basado en huertos o Garden-Based Learning es un término utilizado para aquellas estrategias formativas cuyo recurso didáctico principal es el huerto. Sin embargo, los enfoques teóricos y metodológicos varían enormemente en el panorama educativo, desde aquellos que se centran más en los aspectos experienciales, hasta los que consideran que pueden contribuir a la alfabetización ambiental y el Desarrollo Sostenible (Desmond, Grieshop y Subramaniam 2004).

Existe ya un amplio análisis de programas que utilizan el HE, sobre todo a nivel internacional, (Blair 2009; Desmond, Grieshop y Subramaniam 2004; Dyment 2005; Ozer 2007; Passy, Morris y Reed 2010; Rahm 2002 y William y Dixon 2013) basados tanto en investigaciones cualitativas como cuantitativas.

Blair (2009) realiza una revisión sobre programas de huertos en Estados Unidos y presenta dos conclusiones interesantes respecto a los estudios cuantitativos y cualitativos que analiza: en el análisis de los estudios de tipo cuantitativo concluyen que este tipo de programas actúan positivamente en las áreas de ciencia y tecnología de los alimentos (potencia diversidad de conocimientos relacionados con el medio ambiente, ofrece oportunidades para explorar los fenómenos naturales, mejora la educación nutricional); sin embargo, no pudieron demostrar que hubiese cambios en las actitudes respecto al medio ambiente y comportamiento social. Pero en los estudios cualitativos sí encontraron un alcance más deseable en relación a cambios en los comportamientos sociales y ambientales (alta motivación, orgullo, confianza, unión a la comunidad). Indica también la necesidad de estos programas de la dedicación y participación de diferentes colectivos y la necesidad de una alfabetización sobre agricultura de los docentes.

La Royal Horticultural Society (RHS) realizó un estudio en UK en 2010 de los programas de huertos de la campaña de 2007 (Passy et al. 2010). Este programa ha tratado de unir dos temas claves, el desarrollo sostenible y la vida saludable, dando respuestas a las siguientes preocupaciones: daño ambiental, el aumento de la obesidad, la disminución de la actividad física en la infancia y la creencia de que los escolares y jóvenes están cada vez más distanciados del natural mundo. Los resultados que muestran respecto al ámbito de los valores, es que estos 
programas fomentan comportamientos positivos, mejoras en el bienestar emocional y desarrollo del sentido de la responsabilidad. En relación a los contenidos que se desarrollan, indican tanto aquellos más vinculados al ámbito científico, apuntando que se produce conocimiento y comprensión científica (por ejemplo las estaciones); como los más vinculados a áreas transversales, concretándolo en el ámbito de los alimentos y la salud. También aportan datos respecto a que se produce una mejora en los aspectos aritméticos y el uso de un vocabulario más amplio y mayores habilidades de oratoria, relacionándolo en este caso con otras áreas curriculares.

En el ámbito español, aquellas referencias que se centran en la etapa de infantil, coinciden con las investigaciones anteriores, indicando que el HE es un recurso muy enriquecedor porque «promueve el aprendizaje mediante la experiencia», siendo «un espacio abierto donde alumnos y alumnas están en contacto directo con la tierra, la luz, el agua, las herramientas, las plantas y los animales» (Marín Gil 2015, p. 46).

Para hacernos una idea del alcance de los huertos escolares en el ámbito nacional, se ha realizado un primer mapeo por parte de la empresa Germinando (2018), cuyos datos nos indican que existen unos 2700 centros educativos con huerto escolar, con un alcance potencial de 1.200.000 de alumnos y alumnas. Si centramos la mirada en la comunidad autónoma de Andalucía, podemos encontrar HE que surgen a iniciativa propia de los centros sin vincularse a ningún programa establecido y otros asociados a determinados programas educativos. En este último caso, el programa Ecohuerto acogió a 596 centros, 168.849 alumnado y 923 profesorado durante el curso 2018-2019 (Junta de Andalucía 2019).

\section{Proyecto "Nuestro Huerto" una propuesta didáctica investigativa desde la perspectiva del Decrecimiento}

En primer lugar, se presentan los referentes o perspectivas que fundamentan esta propuesta. Se considera fundamental explicitar tres aspectos esenciales cuando se decide tener un huerto con una función educativa, debido a que la mayoría de las experiencias que se publican no lo hacen. Desmond, Grieshop, y Subramaniam (2004) denominan a alguno de estos aspectos consideraciones operacionales. Es necesario plantearse cual es la finalidad del huerto. Por ejemplo, ¿queremos plantar y conocer las plantas del huerto o queremos conocer la procedencia de nuestros alimentos y el impacto que tienen en el medio y la sociedad? Estas cuestiones estarían relacionadas con la perspectiva socioambiental. También es necesario reflexionar sobre el modelo agroecológico que vamos a utilizar ¿ecológico, convencional, ...? Cada uno de esos modelos lleva consigo una serie de prácticas que se pueden o no se pueden realizar. Y el último aspecto a valorar sería la perspectiva didáctica o la forma de integrar curricularmente el huerto en el aula, es decir, podemos plantearnos ¿realizamos actividades puntuales en el huerto o es el huerto un proyecto de trabajo transversal?

Antes hemos utilizado el término alfabetización ambiental, entendiéndolo como un marco de referencia adecuado para el desarrollo de proyectos educativos. Pero dicha alfabetización se puede ubicar en cualquier de los dos paradigmas actualmente existentes sobre la evolución futura de la humanidad (y del planeta) y sobre las estrategias a emplear para adaptarnos a dicho cambio: el desarrollo sostenible y el decrecimiento. Ambos paradigmas son hipótesis diferentes, pues difieren en su interpretación de los datos científicos actualmente existentes sobre el choque de nuestra civilización con sus límites biofísicos. En la primera hipótesis es factible una reforma del sistema socioeconómico que nos permita adaptarnos al cambio (tal como se sostiene en las propuestas del Green New Deal o en los Objetivos del Desarrollo Sostenible); en la segunda, se parte de la inevitabilidad del decrecimiento, asociado al agotamiento de los recursos, el cambio climático y la disminución de la biodiversidad, de 
forma que solo cabe preparar a la población para que ese decrecimiento sea lo menos lesivo posible, dado que el sistema capitalista sería incapaz de superar su principal contradicción: no es posible mantener el actual desarrollo basado en el crecimiento ilimitado en un planeta finito.

Nuestro proyecto se ubica en la hipótesis decrecentista (Acosta y Ulrich 2018; Casal 2016; Fernández y González 2014; Holmgren 2013; Latouche 2007, 2009 y 2012; Prats, Herrero y Torrego 2016; Taibo 2016; Turiel 2011, 2015 y 2020). De forma que lo que procede es educar a la población para una mejor adaptación a este hecho, entendiendo la alfabetización ambiental dentro de una perspectiva de educación en y para el decrecimiento (García, Rodríguez-Marín, Fernández-Arroyo y Puig 2019; García, Fernández-Arroyo, RodríguezMarín y Puig 2019). Somos conscientes que tal planteamiento aún es minoritario dentro de la comunidad educativa, pero creemos que ello no se debe tanto a que la hipótesis decrecentista sea poco plausible, sino al hecho del fuerte apoyo institucional a las tesis del desarrollo sostenible.

Dentro de este enfoque, los huertos son un recurso muy valioso para reconocer los problemas actuales de la actividad humana, como el cambio climático, el agotamiento de los recursos materiales y energéticos, y la pérdida de biodiversidad. Permiten trabajar el metabolismo circular propio de la biosfera (frente al lineal predominante en nuestro sistema socioeconómico), la idea de que existen límites biofísicos, de que los recursos menguan y que debemos cambiar nuestra organización social para ajustarnos a este decrecimiento.

Desde la perspectiva decrecentista, no nos vale cualquier modelo agroecológico. En nuestro caso, hemos adoptado la permacultura de Holmgren (2013), que como indica el autor va más a allá de la sostenibilidad. El término permacultura (que remite a la idea de agricultura permanente) aparece a mediados de los años 70, para describir un innovador método de diseño del territorio que pretende ser una respuesta a la situación de emergencia planetaria provocada por el modelo de desarrollo socioeconómico basado en el crecimiento ilimitado. La permacultura es más que una tecnología agrícola, entendiéndose como una cierta manera de organizar el espacio y la sociedad, partiendo de tres principios básicos: ajuste adecuado de la actividad humana a los ciclos de la biosfera, la biomímesis (imitación de los procesos naturales), y potenciación de la biodiversidad como un seguro ante la incertidumbre (Holmgren 2013). Con la aplicación de estos principios se busca la máxima eficiencia energética de los sistemas agrícolas, su autosuficiencia, la regeneración de los ecosistemas (sobre todo del ecosistema suelo) y una mejor adaptación al decrecimiento (producir mucho consumiendo menos recursos).

Referido a la perspectiva didáctica, se opta por el modelo de investigación escolar (García y García Pérez 1989), porque es un modelo que se basa principalmente en la capacidad que tenemos las personas parar investigar y en la importancia que tiene para el aprendizaje las ideas y experiencias que posee el alumnado. La actividad educativa debe centrarse en investigaciones realizadas por el alumnado, aprovechando lo que ya saben y ajustando la intervención del profesorado a las características del alumnado, y de sus necesidades en un contexto de menor disponibilidad de recursos.

En resumen, la propuesta que se presenta se basa en una Educación Ambiental en y para el Decrecimiento como perspectiva socioambiental, la Permacultura como modelo agroecológico y la metodología investigativa como perspectiva didáctica. 


\section{Objetivos}

A continuación, mostramos los objetivos de la investigación realizada:

1. Diseñar un proyecto de trabajo para iniciar la alfabetización ambiental en los escolares de Infantil a través del contacto con la naturaleza, utilizando el HE como recurso didáctico.

2. Conocer si los escolares son capaces de plantear soluciones y acciones comunes para dar respuesta a la situación actual de decrecimiento.

3. Explorar los avances que se pueden lograr con los escolares en torno a reconocimiento de la planta como ser vivo, su ciclo vital, las necesidades que limitan su crecimiento y las relaciones entre especies, tomando como base la permacultura.

4. Identificar si la puesta en juego de una estrategia educativa de carácter investigativo es eficaz para el aprendizaje en Educación Infantil.

5. Determinar la eficacia de la implementación del proyecto en el alumnado del primer curso de Educación Infantil.

\section{Metodología}

\section{Participantes y contexto}

Esta experiencia educativa ha contado con una muestra de alumnado de $1^{\circ}$ de Educación Infantil (3 años) del CEIP Olivar de Quinto, una escuela pública situada en la localidad de Dos Hermanas (Sevilla). Dicho centro cuenta con varias zonas dedicadas al huerto: huerto en bancales para cada curso y bosque de alimentos en el patio (figura 1) y huertos en los patios asociados a las clases de infantil (figura 2). La experiencia se desarrolló en el curso 2015/2016, en un aula con 26 sujetos (14 niños y 12 niñas) durante el tercer trimestre.

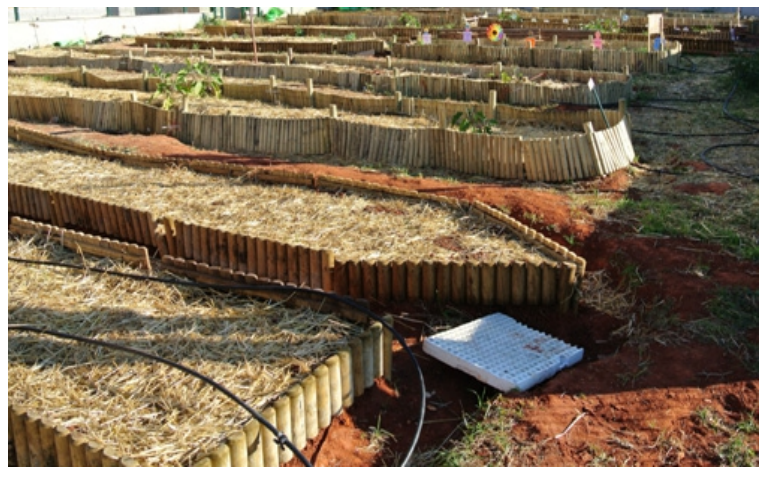

Figura 1. Huerto en bancales para todo el centro.

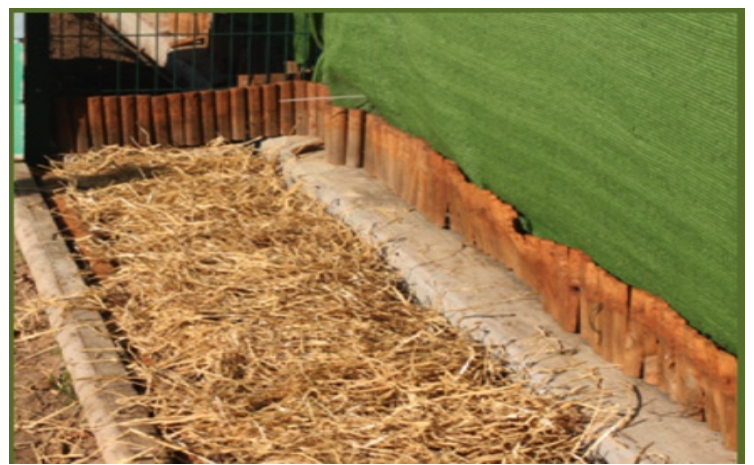

Figura 2. Huerto del patio trasero del Aula de Infantil.

El proyecto fue puesto en práctica en el aula por la tutora del grupo-clase con la ayuda de otra docente que actuó a la vez como investigadora, contando además con el apoyo y asesoramiento de profesorado universitario.

\section{Análisis de la propuesta}

Con el fin de evaluar los efectos de la intervención se utilizaron diferentes instrumentos de evaluación. Por un lado, se recogió información a nivel individual, a través de una serie de dibujos personales, en los que el alumnado tenía que representar los diferentes elementos necesarios para el crecimiento de una planta. Estos dibujos sirvieron como primera toma de contacto con la temática, y su análisis nos sirvió para determinar diferentes niveles (basado en el sistema de categorías creado) dentro del aula. De cada nivel se seleccionó a un sujeto para estudiar la evolución de su aprendizaje a lo largo del proyecto. Se les realizaron entrevistas al inicio y al final de cada problema que fueron grabadas y transcritas por la docente-investigadora. Por otro lado, se utilizaron otras fuentes de 
recogida de información a nivel grupo-clase: asambleas grabadas y transcritas, libro del huerto y cartulinas de cierre, elaborados por el alumnado con la guía de las docentes).

Para el análisis de las respuestas del alumnado, tanto de las entrevistas individuales como de las asambleas, se diseñó un sistema de categorías tomando como referencia investigaciones previas (Cañal 2008), pero formulándolas tras realizar la recogida de datos. Cada nivel sigue una jerarquización en la que las respuestas van desde lo simple (nivel base) a lo complejo (nivel deseable). A continuación, se presenta dicho sistema de categorías:

\section{P1: ¿Qué es una semilla?}

Categoría 1: Grado de conocimiento del concepto de semilla.

I. Indica que la semilla está viva pero no lo justifica, asociando el concepto de semilla con una imagen estática.

II. Indica que la semilla está viva y lo relaciona con algunas necesidades que tiene para crecer, asociando el concepto de semilla con una imagen estática.

III. Indica que la semilla está viva y lo relaciona con alguna etapa del ciclo vital de las plantas (forma parte de un proceso: semilla-planta-flor-fruto).

P2: ¿Cómo crece la planta?

Categoría 1: Grado de conocimiento del ciclo vital de la planta, sus partes y cambios que se producen.

I. No es capaz de identificar ninguna parte de la planta, ni los cambios que en esta se producen.

II. Percibe algunos cambios en las plantas y reconoce algunas partes de ellas, pero no es capaz de explicar sus funciones, ni los procesos que intervienen en su formación.

III. Reconoce muchos de los cambios que se producen en las plantas, relacionando e identificando sus partes y entendiendo algunos procesos necesarios para su formación. Es capaz de explicar algunas funciones de las partes de la planta.

P3: ¿Qué necesita la planta para vivir?

Categoría 1: Grado de conocimiento de los elementos que necesita la planta para vivir.

I. Apenas identifica un elemento (Ej. agua) o ninguno de los que son necesarios para el crecimiento de la planta, sin dar razones por las que este elemento es necesario.

II. Reconoce algunos elementos que la planta necesita para crecer y da razones de para qué sirven.

III. Identifica todos los elementos necesarios para el crecimiento de la planta, muchas de sus características, la medida/cantidad en que son necesarios y las razones de su necesidad, identificando muchas de las funciones de estos elementos.

Categoría 2: Grado de conocimiento de la influencia de la temperatura y las estaciones del año.

I. No reconoce determinados momentos donde hace frío o calor.

II. Identifica momentos de temperaturas bajas o altas, incluso reconoce alguna estación del año.

III. Reconoce las estaciones del año y las temperaturas asociadas a las mismas.

P4: ¿Hay animales en el buerto?

Categoría 1: Grado de conocimiento de los animales del huerto y su papel dentro de él.

I. No reconoce ningún animal presente en el huerto.

II. Reconoce algún animal presente en el huerto, pero no es capaz de percibir su papel e importancia dentro del mismo. 
III. Es capaz de hacer una lista de animales presentes en el huerto e identificar algunas de sus funciones. Empieza a captar la importancia de las relaciones entre seres humanos, plantas y animales. Comprende una cadena de procesos que le llevan a valorar la necesidad de un cambio de alguna actitud respecto a los animales y plantas.

\section{Diseño y descripción de la experiencia}

Para la puesta en marcha de esta experiencia se diseñó el proyecto Nuestro Huerto, compuesto por un total de 20 sesiones de 30-60 minutos cada sesión que se llevó a cabo durante un mes y medio. El proyecto fue diseñado exclusivamente para esta experiencia de investigación, partiendo de las ideas previas del alumnado recogidas en una asamblea de aula, que realizó la docente-investigadora días antes de su incorporación, aprovechando el interés que mostraban por trabajar de nuevo en el huerto y que respondía a las preguntas: ¿qué sabéis?, ¿qué no sabéis?, ¿qué os gustaría saber?...sobre el huerto. En cada sesión se desarrollaron varias actividades, de manera que hubo un total de 27 (cada una de ellas con contenidos de tipo conceptual, actitudinal y procedimental) que abarcaron los cuatro problemas principales del proyecto. A lo largo del mismo se fueron interrelacionando estos problemas, por lo que se trabajaron varios problemas a la vez, lo que hizo que el aprendizaje del alumnado resultara más significativo y espontáneo.

A continuación, se presenta la figura 3 que representa un mapa de problemas con los contenidos de cada problema y la relación entre ellos:

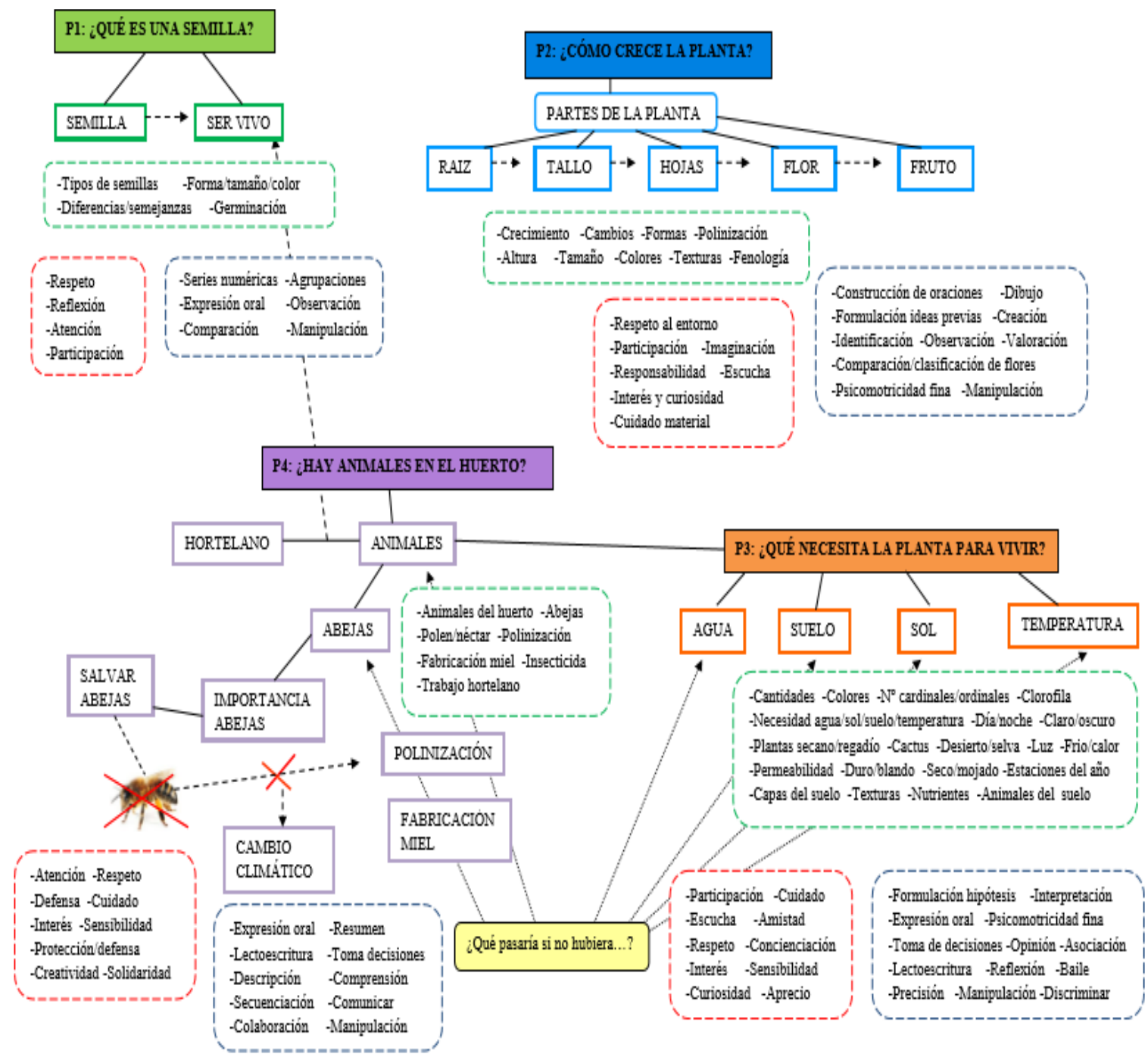

Figura 3. Mapa problemas-contenidos del proyecto. Fuente: Portillo-Guerrero (2016). 
Se ha elegido el problema 4 ¿Hay animales en el huerto?, para su desarrollo de manera resumida en este apartado, presentando en el anexo I las actividades de manera extensa. Además, se puede consultar la propuesta completa de la secuencia del proyecto en el siguiente enlace (https://ecohuertoeducacion.files.wordpress.com/2020/09/secuencia-de-actividadescompleta-3.pdf).

Se comenzó, como en los anteriores problemas, con una lluvia de ideas recogiendo las ideas a modo de listado en el mural correspondiente al problema. Les encantó la idea de buscar animales en el huerto y cada grupo que iba saliendo al patio iba descubriendo un animal nuevo que no se había nombrado antes, lo que enriqueció la lista de animales. Dentro de los animales, les llamó mucho la atención la abeja e investigaron sobre ella, aprendieron el proceso de polinización a través de un cuento, la importancia de su papel en el mundo para la producción de hortalizas (polinización) y fabricación de miel. Fruto de esa concienciación por el cuidado de las abejas, decidieron escribir una carta a Greenpeace contándoles que apoyaban su campaña de salvar a las abejas y que difundirían su mensaje de no usar pesticidas artificiales. La docente-investigadora introdujo el término basándose en esta campaña de la asociación a través de un cuento creado por ella. También recibimos la visita de un experto en huertos al cuál atendieron con mucho interés y preguntaron todas las dudas que tenían. Por último, realizaron una fiesta fin del proyecto, para la cual se disfrazaron con disfraces que habían preparado en sus casas con las familias y también se realizó una asamblea para repasar lo aprendido, realizando el mural correspondiente al problema y parte del libro del huerto.

\section{Resultados}

En respuesta a los objetivos marcados anteriormente, podemos indicar que respecto al objetivo 1, en el apartado de diseño y descripción de la experiencia se ha desarrollado el proyecto, incluyendo los problemas a trabajar y las actividades realizadas, usando de manera activa el huerto escolar de la clase y del centro escolar, como se detallan en las mismas.

Referido al objetivo 2, conocer si el alumnado es capaz de plantear soluciones y acciones comunes para dar respuestas a la situación actual de decrecimiento, se ha trabajado más específicamente en el marco del problema 4 de la secuencia ¿Hay animales en el huerto?, por ejemplo, en relación a la biodiversidad asociada a un huerto (vinculación entre especies animales y vegetales) y al papel de las plantas para la vida en el planeta, relacionado con la alimentación de las personas.

Los resultados obtenidos en la actividad de debate con el hortelano y su preparación, nos demuestran que pueden plantear preguntas diversas, cercanas y lejanas de su contexto. Esto se observa en las preguntas que proponen: ¿de dónde vienen las calabazas? ¿cómo son los animales del huerto? ¿de donde sale el tomate de las albóndigas? ¿hay moscas en el huerto?.Un tema interesante que salió, ha sido el decrecimiento, aprender a vivir mejor con menos. El hortelano les explicó que tenían que ahorrar agua, cuidar el suelo y gastar menos recursos, en general, para que no se agoten estos elementos que necesita la planta y el ser humano para crecer.

Observamos una gran implicación en la creación de la carta que enviaron a Greenpeace para apoyar su campaña y que finalizaba con la siguiente frase a propuesta del alumnado: «Si no hay abejas, no se puede realizar la polinización y entonces no hay frutos y no podemos comer» (ver figura 4). También tuvieron la idea de realizar un dibujo en el que saliera un hombre echando pesticida a las plantas, porque querían explicar que no se debía hacer eso. Así que, que también decidimos mandárselo a la asociación. Cuando la docente informó al alumnado, de la publicación de su carta y la vieron en el blog de la entidad, les impactó bastante que dicha carta fuese publicada por la entidad como agradecimiento . 

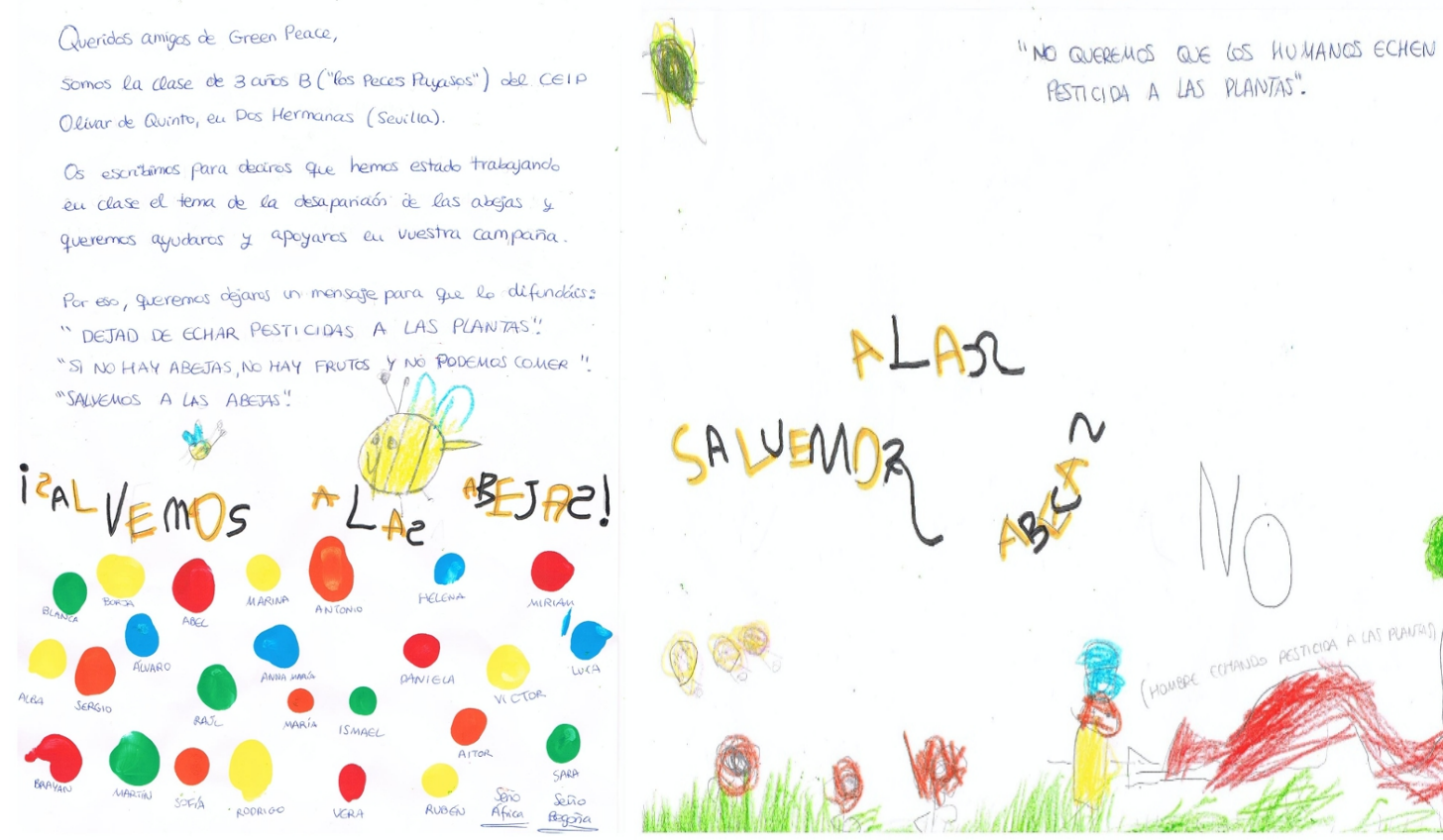

Figura 4. Carta enviada a Greenpeace.

Se ha de destacar que este problema ha sido uno de los que más les gustó al alumnado y en el que llegaron a profundizar en contenidos bastante complejos para su edad, como la polinización y la relación de este proceso con la falta de alimentos. Gracias al cuento de las abejas trabajaron como es este proceso, el papel de los pesticidas, la fabricación de miel, etc. Este recurso sirvió para introducir estos términos, a menudo complejos para el alumnado, que fueron explicados y aclarados por la docente-investigadora. A continuación, en la tabla 1, se muestran las evoluciones observadas tanto en las entrevistas como en las asambleas:

Tabla 1. Resultados de las entrevistas y asambleas respecto al problema 4.

\begin{tabular}{|c|c|c|c|c|c|c|c|c|}
\hline \multirow{2}{*}{$\begin{array}{l}\text { Problemas } \\
\text { del } \\
\text { proyecto }\end{array}$} & \multicolumn{2}{|c|}{ Sujeto 1} & \multicolumn{2}{|c|}{ Sujeto 2} & \multicolumn{2}{|c|}{ Sujeto 3} & \multirow{2}{*}{$\begin{array}{l}\text { Asamblea } \\
\text { Inicial }\end{array}$} & \multirow{2}{*}{$\begin{array}{l}\text { Asamblea } \\
\text { Final }\end{array}$} \\
\hline & Inicial & Final & Inicial & Final & Inicial & Final & & \\
\hline Nivel en P4 & II & III & I & III & II & III & I y II & III \\
\hline
\end{tabular}

Como se observa en la tabla 1, cuando se le preguntaba al alumnado si había animales en el huerto (que fue la pregunta-problema de introducción) algunos se posicionaban en el nivel I, ya que no sabían si había. Otros sabían la presencia de animales (nivel II), siendo los caracoles y las hormigas los más nombrados; y en el momento final todos eran capaces de enumerar al menos 4 animales «Sí, hormigas, saltamontes, mariquitas, abejas, caracoles, mariposas, mosquitos» (sujeto 2). Además, eran capaces de justificar su papel en el huerto y en nuestra alimentación: «la abeja fabrica miel y hace la polinización» y «no hay que echar veneno a las plantas porque se mueren las abejas y no podemos comer» (sujeto 2) «...No se puede echar pesticida a las plantas porque mueren las abejas, y si se mueren las abejas no hay fruto y no podemos comer» (sujeto 3 ). 
En relación al objetivo 3, se presentan los avances que se han logrado con los escolares en torno al reconocimiento de la planta como ser vivo, su ciclo vital, las necesidades que limitan su crecimiento y las relaciones entre especies, tomando como base la permacultura. Estos avances estaban más ligados a los contenidos presentes en los problemas 1, 2 y 3 de la secuencia, que complementan los resultados presentados en Portillo-Guerrero, RodríguezMarín, López-Lozano y Hamed Al-Lal (2018).

Los dibujos que realizaron al inicio de la intervención, con el objeto de identificar qué elementos reconocía el alumnado cómo necesarios para el crecimiento de una planta, nos permitieron identificar los tres niveles presentados en el sistema de categorías relacionados con el problema 3: ¿Qué necesita la planta para vivir?, Categoría 1: Grado de conocimiento de los elementos que necesita la planta para vivir.

Tabla 2. Resultados análisis dibujos individuales.

\begin{tabular}{|c|c|c|}
\hline NIVELES (Problema 3 Cat. 1) & Frecuencia $(\%)$ & Ejemplos \\
\hline \multirow[t]{2}{*}{$\begin{array}{l}\text { I: Apenas identifica un elemento } \\
\text { (Ej: agua) o ninguno de los que } \\
\text { son necesarios para el } \\
\text { crecimiento de la planta, sin dar } \\
\text { razones por las que este } \\
\text { elemento es necesario. }\end{array}$} & $32,00 \%$ & ts \\
\hline & & «Es una semilla con agua» (sujeto 1) \\
\hline \multirow[t]{2}{*}{$\begin{array}{l}\text { II.Reconoce algunos elementos } \\
\text { que la planta necesita para crecer } \\
\text { y da razones de para qué sirven. }\end{array}$} & $44,00 \%$ & \\
\hline & & «Es una flor, el soly círculos» (sujeto 2) \\
\hline \multirow[t]{2}{*}{$\begin{array}{l}\text { III. Identifica todos los } \\
\text { elementos necesarios para el } \\
\text { crecimiento de la planta, muchas } \\
\text { de sus características, la } \\
\text { medida/cantidad en que son } \\
\text { necesarios y las razones de su } \\
\text { necesidad, identificando muchas } \\
\text { de las funciones de estos } \\
\text { elementos. }\end{array}$} & $24,00 \%$ & 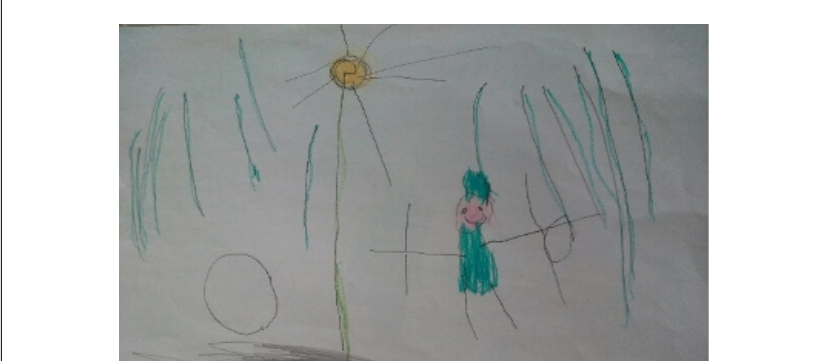 \\
\hline & & $\begin{array}{l}\text { 《Es un jardinero, el sol, una flor, la lluvia, una comadreja y el suelo» } \\
\text { (sujeto 3) }\end{array}$ \\
\hline
\end{tabular}

Se puede percibir que había un mayor número de escolares que se encontraban en un nivel intermedio con un porcentaje de un $44 \%$, seguido del nivel bajo (32\%) y un $24 \%$ pertenecían al nivel alto, nivel III. Entendemos que el nivel madurativo del alumnado influye en estos resultados, de ahí las diferencias en los dibujos mostrados en la tabla 2. 
Posteriormente en las entrevistas individuales, se obtuvieron los resultados recogidos en la tabla 3. Respecto al problema 1, el sujeto 1 pasa del nivel I, no sabía lo que era una semilla: «Un árbol», al nivel III «de la semilla crece una flor». Los sujetos 2 y 3 , se posicionaron en el nivel II al inicio y en el III al final indicando que la semilla «se convierte en una planta». En relación al problema 2 , tanto el sujeto 1 y 2 , se posicionan en el nivel I, dando respuestas poco relacionadas con las preguntas planteadas, y el sujeto 3 se posiciona en el nivel II, ya que es capaz de identificar partes de las plantas «tiene pétalos, flores y el fruto». Todos evolucionan al nivel III en el momento final, con respuestas del tipo: «Raíces, tallo, hojas, fruto (que sale de las flores), Flores (pétalos)...el tallo sirve para aguantar la flor y que no se mueva... el fruto es una fruta que se come y sale de la flor» (sujeto 2). Asimismo, referido al problema 3, el sujeto 1, parte del nivel I y evoluciona hasta el nivel III. Y los sujetos 2 y 3 parten del nivel II hasta alcanzar el nivel III identificando diferentes necesidades de agua y temperatura de algunas plantas.

Tabla 3. Resultados obtenidos de las entrevistas individuales.

\begin{tabular}{|l|l|l|l|l|l|l|}
\hline \multirow{2}{*}{$\begin{array}{l}\text { Problemas del } \\
\text { proyecto }\end{array}$} & \multicolumn{2}{|l|}{ Sujeto 1 } & \multicolumn{2}{l|}{ Sujeto 2 } & \multicolumn{2}{l|}{ Sujeto 3 } \\
\cline { 2 - 7 } & Inicial & Final & Inicial & Final & Inicial & Final \\
\hline Nivel en P1 & I & III & II & III & II & III \\
\hline Nivel en P2 & I & III & I & III & II & III \\
\hline Nivel en P3 & I & III & II & III & II & III \\
\hline
\end{tabular}

Y por último en las asambleas se obtuvieron los resultados que se muestran en la tabla 4.

Tabla 4. Resultados asambleas.

\begin{tabular}{|l|l|l|}
\hline Problemas del Proyecto & Asamblea Inicial & Asamblea Final \\
\hline Nivel en P1 & I y II & III \\
\hline Nivel en P2 & I y II & III \\
\hline Nivel en P3 & I y II & III \\
\hline
\end{tabular}

Con respecto al problema 1, al inicio del mismo, había 2 sujetos que no sabían lo que era una semilla. Esto mismo ocurrió con el concepto de ser vivo, en relación al cual aproximadamente 10 escolares, afirmaban no saberlo. Sin embargo, aparecieron ideas nuevas en la asamblea final, como: «hay diferentes tipos de semillas» «la semilla está viva porque crece...», señalando incluso el crecimiento como función necesaria de los seres vivos. Estos conceptos y los adquiridos en la investigación del problema 1 los reflejaron en las páginas del libro y en la cartulina de cierre del problema que se muestra en la figura 5. 


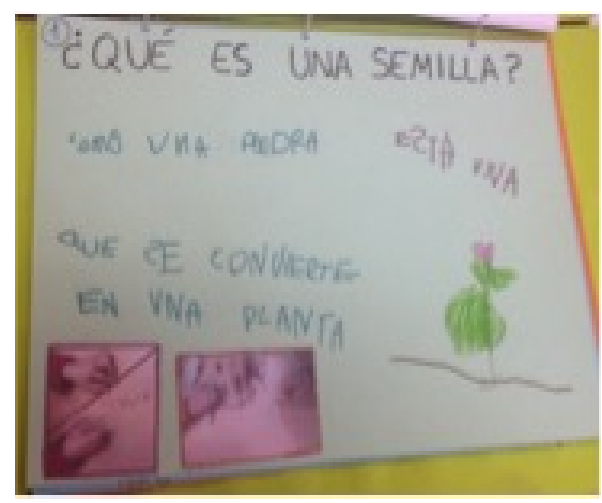

Figura 5. Cartulina para visualizar el cierre pregunta problema 1.

En cuanto al problema 2, la mayoría del alumnado identificaba los pétalos como única parte de la planta. Sin embargo, al final del proyecto, fueron capaces de identificar sus partes y características principales. Al igual que en las entrevistas individuales, en la asamblea global se llegó a un nivel III de conocimiento, evolución y aprendizajes que se reflejaron también en la cartulina de cierre correspondiente (ver figura 6).

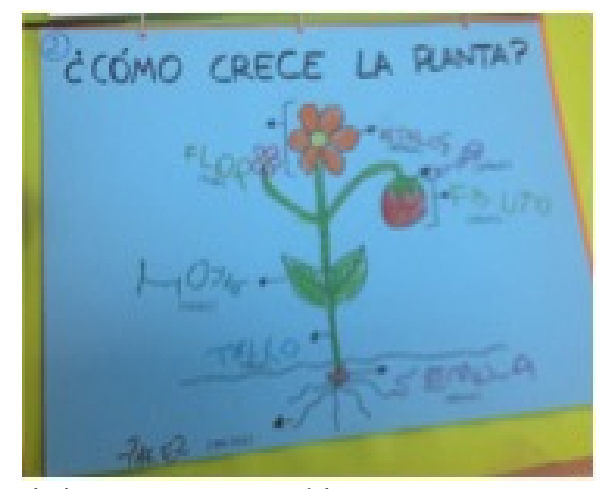

Figura 6. Cartulina para visualizar el cierre pregunta problema 2.

Respecto al problema 3, también se apreció la evolución de la clase. Al principio del proyecto, en asamblea, identificaban más elementos necesarios para el crecimiento de la planta que a nivel individual, pero no sabían en qué proporciones los necesitaba («mucha agua», "poca agua»). Al final del problema aprendieron a relativizar la cantidad de agua que necesitaba la planta, dependiendo de la frecuencia de riego y del tipo de planta. Esto lo reflejaron en una de las páginas del libro que se muestra en la figura 7.

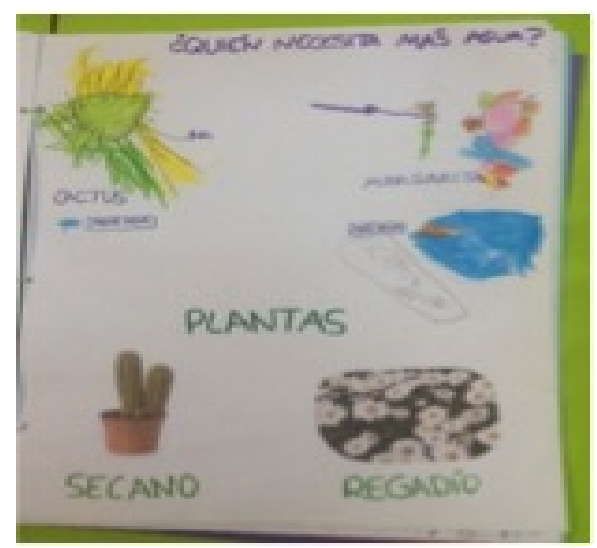

Figura 7. Página del libro diferencias entre plantas (cantidad de agua).

Otra particularidad extraída de la asamblea del grupo-clase es que al principio no reconocían las estaciones y afirmaban que estas eran «frío, calor, navidad, vacaciones...». Esta idea cambió 
y al final fueron capaces de nombrar las 4 estaciones, relacionándolas con la necesidad de una temperatura determinada para las plantas y a su vez con las distintas plantaciones a lo largo del año.

Respecto a los dos últimos objetivos que nos marcábamos con esta experiencia: identificar si la puesta en juego de una estrategia educativa de carácter investigativo es eficaz para el aprendizaje en Educación Infantil y determinar la eficacia de la implementación del proyecto en el alumnado del primer curso de Educación Infantil. Podemos decir que como se observa en los datos, aunque el alumnado partía de niveles de conocimiento diferentes, dado que presentan niveles madurativos distintos, todos han llegado al nivel de conocimiento más complejo propuesto en el proyecto. Esto nos lleva a entender que la metodología basada en la investigación utilizada ha sido adecuada y se ha adaptado a la diversidad del alumnado del aula. Asimismo, los resultados alcanzados nos demuestran que, aunque hay contenidos que no suelen trabajarse en Educación Infantil pueden tratarse en esta etapa, ya que no se tuvo que eliminar ninguna actividad por el tipo de contenidos que abordaban.

\section{Conclusiones}

Los resultados obtenidos nos llevan a valorar de forma positiva la experiencia realizada, manifestando el cumplimiento de los objetivos propuestos. Así pues, el huerto ha resultado ser un recurso de gran utilidad para iniciar la alfabetización ambiental de los escolares de Educación Infantil, a través del contacto con la naturaleza. Las actitudes de cuidado y preocupación por la naturaleza han aumentado, al tiempo que se ha observado una evolución de los aprendizajes en relación a contenidos ambientales.

El proyecto realizado ha permitido a los más pequeños acercarse a la situación real de nuestro planeta y buscar posibles soluciones que implicaban la realización de acciones comunes. Tomar conciencia de la situación de emergencia planetaria en la que nos encontramos y sentirnos responsables de la búsqueda de posibles soluciones, era un objetivo de este proyecto, que se ha visto con creces cubierto al proponer el propio alumnado una acción concreta para una campaña de concienciación sobre las abejas de una asociación.

Respecto a la exploración en los avances que se pueden lograr en los aprendizajes, coinciden nuestros resultados con los de Cañal (2008) en la identificación del agua como el principal componente del material necesario para que crezca una planta (por la importancia del riego diario). En relación con las concepciones que tienen del suelo, nos sorprende como escolares de solo 3 años identifican en él tanto elementos vivos como inertes, aspecto que se observa en investigaciones con estudiantes más mayores (Battista, Prieto, Blanco y González 2001). Sin embargo, no se han podido trabajar ni los nutrientes del suelo, ni la nutrición de las plantas debido a la complejidad de estos conceptos para su desarrollo cognitivo. La propuesta ha servido para adquirir habilidades manipulativas como las descritas por Fernández y Rodríguez (2006). El alumnado ha trabajado con los diversos materiales ofrecidos, utilizando elementos pequeños como las semillas, midiendo cantidades de agua y observando diferencias en los tipos de tierra y elementos del suelo (Portillo Guerrero, Rodríguez-Marín, López-Lozano y Hamed 2018).

Podemos indicar que este proyecto ha resultado eficaz, puesto que nos ha permitido cumplir con todos los objetivos propuestos, sin embargo, consideramos que se pueden plantear algunas propuestas de mejora: mayor implicación de las familias, mayor dedicación al trabajo fuera del aula y el uso de materiales específicos para infantil, que debido a que existen pocos se tenían que diseñar. 
Finalmente, esta experiencia nos ha brindado la oportunidad de aplicar una estrategia educativa de carácter investigativo, donde el alumnado se convierte en el centro del proceso de aprendizaje, desarrollando un papel activo de construcción del conocimiento. Los escolares de 3 años, han demostrado una gran capacidad para la investigación, pues su constante estado de curiosidad la favorece. Así, han sido capaces de plantear hipótesis, contrastarlas, reformular ideas y trabajar de manera conjunta para dar respuesta a cuestiones que les inquietaban. Podemos concluir que la utilización del huerto escolar como eje central de esta propuesta didáctica basada en el modelo de investigación escolar ha sido un recurso que ha favorecido la alfabetización ambiental del alumnado participante. En este sentido, animamos a los centros educativos a implicarse en la utilización de los huertos que poseen, dando un paso más en los contenidos que se trabajan para poder contribuir a aspectos tan relevantes como la resiliencia de la población, tan necesarios en situaciones de emergencia como la que estamos viviendo.

\section{Agradecimientos}

Esta investigación se ha podido llevar a cabo gracias a la colaboración del CEIP Olivar de Quinto (Dos Hermanas, Sevilla) y a las docentes de la etapa de Educación Infantil.

\section{Referencias bibliográficas}

Acosta A., Ulrich B. (2018) Salidas del laberinto capitalista. Decrecimiento y postextractivismo. Barcelona: Icaria Editorial.

Álvarez-García O., Sureda-Negre J., Comas-Forgas R. (2018) Evaluación de las competencias ambientales del profesorado de primaria en formación inicial: estudio de caso. Enseñanza de Las Ciencias. Revista de Investigación y Experiencias Didácticas, 36(1), 117-141.

Battista T.; Prieto T.; Blanco A., González F. (2001) Actividades para la iniciación del concepto de suelo. Alambique: Didáctica de las ciencias experimentales, 30, 55-66.

Blair D. (2009) The child in the garden: An evaluative review of the benefits of school gardening. Journal of Environmental Education, 40(2), 15-38.

Cañal P. (2006) La alfabetización científica en la infancia. Pedro Cañal de León Universidad de Sevilla. Aula de Infantil, 33, 5-9.

Cañal P. (2008) Proyecto Curricular Investigando Nuestro Mundo (6-12). Investigando los seres vivos. Sevilla: Díada.

Casal Lodeiro M. (2016) La izquierda ante el colapso de la civilización industrial. Madrid: La Oveja Roja.

Collado S. (2012) Experiencia infantil en la naturaleza. Efectos sobre el bienestar y las actitudes ambientales en la infancia. Tesis doctoral.

Desmond D., Grieshop J., Subramaniam A. (2004) Revisiting garden-based learning in basic education. International Institute for Educational Planning. Food and Agriculture Organization of the United Nations. Recuperado de www.unesco.org/iiep

Dyment J. E. (2005) Green School Grounds as Sites for Outdoor Learning: Barriers and Opportunities. International Research in Geographical and Environmental Education, 14(1), 28 45.

Eshach H., Fried M. N. (2005) Should science be taught in early childhood? Journal of Science Education and Technology, 14(3), 315-336.

Eshach H. (2006) Science literacy in primary schools and pre-school, 2006-2008. https://doi.org/10.1007/1-4020-4674-X 
Fernández R., González L. (2014) En la espiral de la energía. Madrid: Libros en Acción. Baladre. Frank.

Fernández R., Rodríguez L. (2006) Los pequeños de cuatro años en el rincón de ciencias: qué ven y qué dicen sobre el nacimiento de las plantas. Alambique, 49, 105-113.

Fjortoft I. (2001) The Natural Environment as a Playground for Children: The Impact of Outdoor Play Activities in Pre-Primary School Children. Early Childhood Education Journal, 29, 111-117.

García-Carmona A., Criado, A. M., Cañal P. (2014) Alfabetización científica en la etapa 3-6 años: Un análisis de la regulación estatal de enseñanzas mínimas. Enseñanza de las Ciencias, 32(2), 131-149.

García J. E. (2006) Educación ambiental y alfabetización científica: argumentos para el debate. Investigación en la Escuela, 20(60), 7-19.

García J.E., García Pérez, F. F. (1989) Aprender investigando: una propuesta metodológica basada en la investigación. Sevilla: Diada Editora.

García J.E., Rodríguez-Marín F., Fernández-Arroyo J. , Puig M. (2019) La educación científica ante el reto del decrecimiento. Alambique: Didáctica de las ciencias experimentales, 95, 47-52.

García J.E., Fernández-Arroyo J., Rodríguez-Marín F., Puig M.(2019) Más allá de la sostenibilidad: por una educación ambiental que incremente la resiliencia de la población ante el decrecimiento/colapso. Revista de Educación Ambiental y Sostenibilidad, 1(1) 1101. doi: 10.25267/Rev_educ_ambient_sostenibilidad.2019.v1.i1.1101

Germinando (2018) Germinando espacio agroecológico. http://germinando.es/mapeo-estatal-dehuertos-escolares/

Gómez-Montilla C. , Ruiz-Gallardo J. R. (2016) El rincón de la ciencia y la actitud hacia las ciencias en Educación Infantil. Revista Eureka sobre Enseñanza y Divulgación de las Ciencias, 13 (3), 643-666

Guzmán-Cruz M., García-Carmona A., Criado A. M. (2017) Aprendiendo sobre los cambios de estado en educación infantil mediante secuencias de pregunta-prediccióncomprobación experimental. Enseñanza de Las Ciencias, 35 (3),175-193.

Holmgren D. (2013) Permacultura: principios y senderos más allá de la sustentabilidad. Argentina: Kaicron

Junta de Andalucía (2019) Aldea Programa de Educación Ambiental para la comunidad educativa. Catálogo curso 2019-20. Sevilla: Consejería de Medio Ambiente y Ordenación del Territorio y Consejería de Educación.

Latouche S. (2007) Sobrevivir al desarrollo. Barcelona: Icaria Editorial.

Latouche S. (2009) Pequeño tratado del decrecimiento sereno. Barcelona: Icaria Editorial.

Latouche S. (2012) La sociedad de la abundancia frugal. Barcelona: Icaria Editorial.

Louv R. (2005) Last child in the Woods: saving our children from nature-deficit disorder. New York: Workman Publishing Company.

McCurdy L., Winterbottom K., Mehta S., Roberts, J. (2010) Using nature and outdoor activity to improve children's health. Current problems in pediatric and adoles- cent health care. 40(5) 102-117. 
Marín Gil J. (2015) El huerto escolar: mágica fuente de conocimiento y sabiduría. Aula, 239, 44-47.

Mazas B., Hervas A. (2018) Los niños y las niñas de infantil piensan, actúan y hablan sobre el comportamiento del aire y del agua, Enseñanza de las Ciencias, 36(1), 163-180.

Mergen B. (2003) Review essay: Children and nature in history. Environmental History, 8, 643669.

NLI (The Natural Learning Initiative) (2012) Benefits of connecting children with nature: Why naturalize outdoor learning environments. https://naturalearning.org/sites/default/files/Benefits of Connecting Children with Nature_InfoSheet.pdf

Ozer E. J. (2007) The effects of school gardens on students and schools: Conceptualization and considerations for maximizing healthy development. Health Education and Behavior, 34(6), 846-863.

Palmer S. (2006) Toxic Childhood: How the Modern World is Damaging our Children and What We can Do About It. London: Orion.

Passy R., Morris M., Reed, F. (2010) Impact of school gardening on learning. Primary Care.

Poppell K., Monroe M. C. (2017) Why is Exposure to Nature Important in Early Childhood? Recuperado de http://edis.ifas.ufl.edu/fr394

Portillo Guerrero, M. de A. (2016). El huerto escolar como instrumento de cambio social dentro del trabajo por proyectos. Trabajo Fin de Grado. Universidad de Sevilla

Portillo Guerrero M. de A., Rodríguez-Marín F., López-Lozano L., Hamed Al-Lal S. (2018) $¿$ Que necesita una planta para vivir? Una propuesta didáctica investigativa en Infantil. En C. Martínez Losada y S. García Barros (Eds.), 28 Encuentros de Didáctica de las Ciencias Experimentales: Iluminando el cambio educativo (pp. 245-250). A Coruña: Universidade da Coruña, Servizo de Publicacións. https://doi.org/10.17345/comeduc199253

Prats S., Herrero Y., Torrego A. (2016) La Gran Encrucijada. Barcelona: Libros en Acción /Icaria

Prince C. (2010) Sowing the seeds: education for sustainability within the early years curriculum. European Early Childhood Education Research Journal, 18:3, 423-434, DOI: 10.1080/1350293X.2010.500082

Rahm J. (2002) Emergent learning opportunities in an inner-city youth gardening program. Journal of Research in Science Teaching, 39(2), 164-184.

Taibo C. (2016) El colapso. Madrid: Los Libros de la Catarata.

Torres-Porras J., Alcántara J., Arrebola J. C., Rubio, S. J., Mora, M. (2017) Trabajando el acercamiento a la naturaleza de los niños y niñas en el Grado de Educación Infantil. Crucial en la sociedad actual. Eureka Sobre Enseñanza y Divulgación de Las Ciencias,14(1), 258-270.

Trainer T. (2017) La vía de la simplicidad. Hacia un mundo sostenible y justo. Madrid: Trotta.

Turiel A. (2011) Por qué se despilfarra tanto. Blog The oil crash

Turiel A. (2015) El contexto de nuestro colapso. Blog The oil crash. 
Turiel A (2020) Petrocalipsis: Crisis energética global y cómo (no) la vamos a solucionar. Madrid: Alfabeto.

Williams D. R., \& Dixon P. S. (2013) Impact of Garden-Based Learning on Academic Outcomes in Schools. Review of Educational Research, 83(2), 211-235. 
Anexo I. Descripción actividades Problema 4.

\begin{tabular}{|c|c|}
\hline \multirow[t]{3}{*}{ A1 (Ideas Previas) } & Título: ¿Por qué las flores tienen tantos colores? \\
\hline & $\begin{array}{l}\text { Objetivos:Reflexionar sobre las preguntas concretas } \\
\text { respetando ideas de los demás compañeros }\end{array}$ \\
\hline & $\begin{array}{l}\text { Descripción: En asamblea se dialoga haciendo una síntesis de } \\
\text { las características de las flores que habían sido trabajadas en } \\
\text { actividades anteriores. En este diálogo la docente lanza la } \\
\text { pregunta ¿Por qué tendrán tantos colores las flores? Entre } \\
\text { todos se plantean hipótesis, surgiendo entre ellas la idea de que } \\
\text { fuera para atraer a los animales. La docente explica esto con } \\
\text { más detalles y comienza de esta forma el problema } 4 \text {. }\end{array}$ \\
\hline \multirow[t]{3}{*}{ A2 (Actividad de contraste) } & Título: ¡Vamos a buscar animales al huerto! \\
\hline & $\begin{array}{l}\text { Objetivos: Indagar en el huerto sobre la existencia de animales, } \\
\text { respetando y cuidando los elementos del entorno más cercano } \\
\text { y valorar la importancia de las flores }\end{array}$ \\
\hline & $\begin{array}{l}\text { Descripción: Tras una lluvia de ideas en la que los niños/as } \\
\text { fueron diciendo los animales que pensaban que iban a } \\
\text { encontrar en el huerto, salieron por grupos a observar } \\
\text { directamente el huerto en busca de animales. Cuando un } \\
\text { grupo llegaba al aula, anotaba en la pizarra con la ayuda de la } \\
\text { docente aquellos animales que había podido ver. Al final de la } \\
\text { sesión, se leyó la lista completa y se dialogó sobre cómo es y } \\
\text { qué hace cada uno de los animales encontrados. } \\
\text { Los animales encontrados fueron incluidos en el libro del } \\
\text { huerto, escribiendo los propios niños/as sus nombres. }\end{array}$ \\
\hline \multirow[t]{3}{*}{ A3 (Actividad de contraste) } & Título: Las abejas \\
\hline & $\begin{array}{l}\text { Objetivos: Ordenar cronológicamente una secuencia de } \\
\text { imágenes y entender el proceso de polinización y de } \\
\text { fabricación de miel. }\end{array}$ \\
\hline & $\begin{array}{l}\text { Descripción: La docente mostró en la pantalla digital imágenes } \\
\text { de abejas. Los niños/as fueron comentando lo que veían y } \\
\text { dialogando sobre las características de estos insectos. En este } \\
\text { diálogo surgió la idea de que las abejas fabrican miel, pero } \\
\text { ninguno sabía cómo era el proceso. Con ayuda de unas } \\
\text { ilustraciones que organizaban el proceso, la docente mostró } \\
\text { una secuencia de } 4 \text { pasos. Finalizada la explicación, se } \\
\text { repartieron las ilustraciones entre los niños/as y ellos mismos } \\
\text { fueron ordenando el proceso. } \\
\text { Se siguió el mismo modelo para el proceso de polinización. } \\
\text { Para finalizar, los niños/as incluyeron las secuencias ordenadas } \\
\text { en el libro del huerto. }\end{array}$ \\
\hline \multirow[t]{3}{*}{ A4 (Actividad de contraste) } & Título: ¿Qué pasaría si no hubiera abejas? \\
\hline & $\begin{array}{l}\text { Objetivos: Acercarse a la lectoescritura y adquirir el valor de } \\
\text { cuidado y protección de las abejas. }\end{array}$ \\
\hline & $\begin{array}{l}\text { Descripción: Se realizó la lectura de cuento elaborado por la } \\
\text { docente/investigadora titulado "Un problema en la colmena". } \\
\text { En el cuento, las abejas, para solucionar la muerte de sus } \\
\text { compañeras, llamaban a un amigo hortelano que las ayudó. } \\
\text { Este envió una carta al presidente para que prohibiera el uso } \\
\text { de pesticidas y las abejas no murieran. } \\
\text { Finalizado el cuento se dialogó sobre las ideas principales del } \\
\text { mismo. }\end{array}$ \\
\hline
\end{tabular}


Anexo I. Continuación.

\begin{tabular}{|c|c|}
\hline \multirow[t]{3}{*}{ A5 (Actividad de contraste) } & Título: ¿Qué podemos hacer para cuidar a las abejas? \\
\hline & $\begin{array}{l}\text { Objetivos: Aprender propuestas alternativas para no dañar el } \\
\text { medio ambiente, fabricar insecticida natural y escribir carta de } \\
\text { concienciación a Greenpeace. }\end{array}$ \\
\hline & $\begin{array}{l}\text { Descripción: Al hilo del cuento, la docente/investigadora } \\
\text { plantea que había leído una noticia similar a lo ocurrido en el } \\
\text { cuento en la prensa y que, por tanto, estaba pasando en la } \\
\text { realidad. Rápidamente a uno de los niños se le ocurrió la idea } \\
\text { de escribir una carta, por lo que la docente/investigadora } \\
\text { explicó que había un grupo de personas que se encargaban de } \\
\text { salvar a las abejas, por lo que podían escribirles una carta para } \\
\text { decirles que los queríamos ayudar y transmitirles su mensaje: } \\
\text { "No echéis pesticida a las plantas", "si no hay abejas, no hay } \\
\text { frutos y no podemos comer", "salvemos a las abejas". Otro } \\
\text { niño, tuvo la idea de realizar un dibujo en el que saliera un } \\
\text { hombre echando pesticida a las plantas, porque quería explicar } \\
\text { que no se debía hacer eso. Es por esto, que también se decidió } \\
\text { mandárselo a la asociación. } \\
\text { De este modo, se elaboró una carta entre todos, que fue } \\
\text { escribiendo la docente/investigadora. Al final de la carta, } \\
\text { todos firmaron con su huella y algunos escribieron el lema } \\
\text { principal. Dicha carta junto con el dibujo del hombre echando } \\
\text { pesticidas fue enviado a Green Peace. }\end{array}$ \\
\hline \multirow[t]{3}{*}{ A6 (Actividad de contraste) } & Título: La visita del hortelano \\
\hline & $\begin{array}{l}\text { Objetivos: Atender a las explicaciones de nuestro visitante y } \\
\text { saber plantear preguntas y expresarlas oralmente. }\end{array}$ \\
\hline & $\begin{array}{l}\text { Descripción: Durante la visita del hortelano los niños/as } \\
\text { fueron realizando las preguntas que días antes habían } \\
\text { preparado y que docente había dejado anotadas. El hortelano } \\
\text { fue respondiendo a las preguntas y los niños/as fueron } \\
\text { dialogando de manera fluida sobre aquello que les llamaba la } \\
\text { atención. En este diálogo, el hortelano insistió mucho en } \\
\text { algunas ideas directamente vinculadas con el decrecimiento, } \\
\text { tratando de acercar a los más pequeños a una perspectiva } \\
\text { compleja, de la que les destacó la necesidad de gastar menos } \\
\text { recursos y cuidar lo que tenemos para poder seguir viviendo } \\
\text { en este planeta. }\end{array}$ \\
\hline \multirow{3}{*}{ A7 (Actividad de cierre y recapitulación) } & Título: ¿Qué hemos aprendido? \\
\hline & $\begin{array}{l}\text { Objetivos: Disfrazarse de un elemento visto en el proyecto y } \\
\text { recapitular y resumir lo aprendido en el proyecto }\end{array}$ \\
\hline & $\begin{array}{l}\text { Descripción: Los niños/as junto a sus familias, habían } \\
\text { realizado disfraces en casa que llevaron ese día. Cada uno/a } \\
\text { iba disfrazado de algo que había visto o aprendido a lo largo } \\
\text { del proyecto. Se hicieron fotos en el mural ya terminado del } \\
\text { proyecto y para concluir, se realizó una asamblea final en la } \\
\text { que fueron recordando lo aprendido en cada uno de los } \\
\text { problemas a investigar viendo las respuestas que se habían ido } \\
\text { dando a los mismos. }\end{array}$ \\
\hline
\end{tabular}

